\title{
Comparando Estruturas Sociais no Rio de Janeiro e em São Paulo
}

\author{
Eduardo Marques \\ Celi Scalon \\ Maria Aparecida Oliveira
}

\begin{abstract}
presente artigo apresenta resultados recentes de pesquisa sobre a distribuição da estrutura social nas metrópoles carioca e paulistana de forma comparada. São utilizadas aqui metodologias específicas dos estudos de estratificação social: as classes EGP, desenvolvidas por Erikson, Goldthorpe e Portocarrero, e o Índice Internacional de Status Socioeconômico Ocupacional (ISEI) ${ }^{1}$, calculados por Scalon (2006), para caracterizar a estrutura socioocupacional nas duas cidades em 2000, partindo de dados do Censo Demográfico do Instituto Brasileiro de Geografia e Estatística - IBGE. As informações utilizadas dizem respeito às áreas de ponderação do Censo, unidade de maior desagregação para a qual são publicados os dados da amostra. A partir delas, comparamos as estruturas existentes, assim como, por meio da utilização dos Sistemas de Informações Geográficas, espacializamos as categorias, investigando as duas conformações metropolitanas e as estruturas de segregação presentes nos dois casos. Os resultados confirmam os resultados de trabalhos anteriores para o início da década de 1990, mas acrescentam novas informações a respeito das estruturas metropolitanas em tela.
\end{abstract}

O artigo está organizado em três partes, além desta introdução e da conclusão. Inicialmente, recuperamos rapidamente o tratamento dado ao tema na literatura nacional desde os anos 1970. Em seguida, apresentamos a metodologia utilizada, para na terceira parte analisarmos

DADOS - Revista de Ciências Sociais, Rio de Janeiro, Vol. 51, n-1, 2008, pp. 215 a 238. 
comparativamente as duas estruturas. A quarta e última seção espacializa as informações e compara os padrões de segregação. Retomamos os principais achados ao final, à guisa de conclusão.

\section{O DEBATE DA LITERATURA SOBRE FORMA URBANA, ESTRUTURA SOCIAL NO ESPAÇO}

A discussão sobre a distribuição espacial da estrutura social em cidades brasileiras é já bastante antiga e consolidada, remontando ao início da sociologia e dos estudos urbanos no Brasil nos anos 1970. Na verdade, essas preocupações já estavam presentes nas críticas à teoria da marginalidade, que acabariam por desembocar em nossos primeiros estudos urbanos, dada a centralidade do mercado de trabalho (ou das classes) nos debates (Maricato, 1977; Camargo, 1976), e persistiu quando o principal elemento integrador dos debates era a categoria periferia $^{2}$.

Embora o registro das discussões nesse momento fosse ensaístico, e principalmente ancorado teoricamente no marxismo, já se faziam presentes fortes premissas com relação à distribuição dos grupos sociais em nossos espaços metropolitanos. Nesse registro, a estruturação social em nossas cidades foi tratada tanto de maneira indireta, associada à maneira como se pensava a produção do espaço (Bonduki e Rolnik, 1982), como de forma mais explícita, derivando conformações urbanas de interpretações sociológicas macro sobre o funcionamento de nossas cidades (Santos e Bronstein, 1978). De maneira geral, as análises descreviam nossas grandes cidades como marcadas por formas relativamente radiais e concêntricas de características positivas decrescentes do centro para as periferias. Essas últimas consistiriam de vastas extensões territoriais em homogêneas (na pobreza e na precariedade), onde residiriam os responsáveis por fazer girar a "maquinaria econômica" (Kowarick, 1979:43), submetidos a condições muito precárias de vida, em habitações autoconstruídas em áreas irregulares ou ilegais e desconsideradas pela ação do Estado.

Outros trabalhos também trataram da questão naquele momento, mas em um registro mais empiricamente embasado e focado centralmente na distribuição dos grupos sociais na cidade. Nessa tradição, temos tanto estudos de cunho mais aplicado, orientados para o apoio a políticas públicas (Brasileiro, 1976; Taschner e Bógus, 1999), como com objetivos mais analíticos, como Vetter (1981), que se dedicou intensamente 
a analisar a distribuição dos grupos sociais, equipamentos coletivos e políticas no espaço carioca.

Embora essa preocupação tenha estado sempre presente ao longo das décadas seguintes, a centralidade da estrutura social e das periferias foi deslocada por outros debates, em parte impulsionada pela própria dinâmica do campo, em parte influenciada pelas mudanças nas agendas políticas nacionais com a redemocratização (Kowarick, 2000). No início dos anos 1990, o tema voltou a se colocar com a publicação de trabalhos como Sposati (1996), que o reintroduziram em um novo patamar metodológico com a utilização de análise quantitativa de dados socioeconômicos para analisar quadros de desigualdade no espaço, lançando mão da espacialização de grupos sociais como estratégia analítica. Desde então vários outros trabalhos foram produzidos com essa mesma temática, como produtos de estratégias analíticas e de sofisticações metodológicas distintas. Em uma linha comparativa ampla e recuperando a história de construção de várias metrópoles brasileiras, vem a pesquisa de Villaça (1998). Em um esforço de grande porte para traduzir as categorias socioocupacionais do debate sociológico francês, diversos autores associados ao Observatório das Metrópoles do Instituto de Pesquisa e Planejamento Urbano e Regional - IPPUR vêm analisando (e comparando) as estruturas de cidades como Rio de Janeiro, São Paulo, Belo Horizonte, Salvador e Paris (Taschner e Bógus, 1999 e 2000; Ribeiro, 2002; Ribeiro e Lago, 2000; Lago, 2002; Mendonça, 2003; Carvalho, Souza e Pereira, 2004; Ribeiro e Preteceille, 1999). Em uma estratégia distinta, que utilizou diretamente com as variáveis censitárias, sem lançar mão da construção de categorias sociais preestabelecidas e construindo classificações socioeconômicas a partir das distribuições empíricas dos indicadores, inscrevem-se estudos como Marques e Bitar (2002) e CEM (2004). Esse conjunto de trabalhos associou-se a outro, que analisou as conseqüências de tais padrões de segregação em diversas dimensões sociais, como Marques e Torres (2005) e Bichir (2006).

Scalon (2006), por outro lado, utilizou para as áreas de ponderação de Rio de Janeiro e São Paulo as categorias EGP a partir das informações censitárias, espacializando as categorias nas duas cidades e avaliando a estrutura das ocupações em São Paulo ao longo da década de 1990, e demonstrando uma grande permanência, exceto pela inserção das mulheres nas ocupações. As informações analisadas nas seções que se seguem dão continuidade a essa linha de análise, aprofundando o estudo 
comparado da estrutura social das duas cidades e investigando as similaridades e diferenças de suas configurações urbanas e de segregação.

Desse modo, combinamos a discussão sobre estrutura social e estrutura urbana com argumentos próprios da literatura sobre estratificação e classe, entendendo que a estrutura de classes está intimamente vinculada à forma de organização do espaço urbano. Para Bourdieu (1989), as classes são formações de agentes que ocupam posições semelhantes no espaço social, o que implica uma maior probabilidade de adotar atitudes e interesses semelhantes. Nesse sentido, a estrutura social pode ser entendida em termos de topologia social, uma vez que é no espaço social que se distribuem essas posições e se estabelecem as distâncias que as separam. Segundo ele, "o que existe é um espaço de relações, que é tão real como um espaço geográfico, no qual as mudanças de lugar se pagam em trabalho, em esforço e, sobretudo, em tempo [...]. Dessa forma, nada mais lógico que tentar relacionar o 'espaço social' com o 'espaço geográfico'" (ibidem:137).

\section{A METODOLOGIA EMPREGADA}

Neste artigo, serão utilizadas as classes EGP e o Índice ISEI para a construção de uma interpretação a respeito da estrutura social nas duas cidades em questão.

As classes EGP são o esquema de classes desenvolvido por Erikson, Goldthorpe e Portocarrero (1979), incorporando as iniciais dos autores. Embora o debate inclua um amplo conjunto de classificações (Scalon, 1999), esse esquema foi escolhido por ser internacionalmente utilizado, o que amplifica as possíveis comparações a serem desenvolvidas posteriormente. Em termos empíricos, mostrou-se adequado e robusto para o estudo em questão. Uma importante qualidade do esquema EGP é que ele distingue posições no mercado de trabalho mas também reflete níveis de qualificação e diferenças setoriais. A questão remonta à longa tradição na literatura inglesa, que já pode ser reconhecida no estudo de Lockwood, em 1958: The Blackcoated Worker. Tomando como base a idéia de classe weberiana, Lockwood define três conceitos que ganharam dimensão expressiva nos estudos de classes: (a) situação de mercado, que indica a posição econômica, em termos de probabilidade de ser inserido no mercado de trabalho; treinamento, qualificações e experiências oferecidas no mercado; grau de segurança (esta- 
bilidade); oportunidade de mobilidade ascendente e de recompensas materiais como salário, renda ou outras; (b) situação de trabalho, que inclui o conjunto de relações nas quais o indivíduo está envolvido em seu emprego em virtude da posição que ocupa na divisão do trabalho; e (c) situação de status, que corresponde à posição na hierarquia de prestígio da sociedade como um todo.

Esses três conceitos estão inseridos na classificação EGP, que tem como elementos básicos para a agregação de categorias ocupacionais a posição de mercado, ou seja, a posição na estrutura econômica e a posição de trabalho, entendida como a posição nas relações de produção. Assim, os conceitos de classe, estrato e ocupação entrecruzam-se nessa análise dada a própria definição de classe adotada aqui, qual seja: situação de classe como situação de mercado e situação de trabalho. Neste estudo, optamos por utilizar a classificação com seis classes agregadas, englobando: I. Profissionais e Administradores; II. Trabalhadores Não-Manuais de Rotina; III. Pequenos Proprietários; IV. Trabalhadores Manuais Qualificados; V. Trabalhadores Manuais Não-Qualificados; VI. Rural. Essa agregação nos dá pouco detalhamento da estrutura social, informa-nos de maneira bastante eloqüente sobre as características gerais dessa estrutura, adequando-se bastante a nossos objetivos aqui. $\mathrm{O}$ uso de classes mais abertas daria acesso a maior detalhamento, mas perderíamos visibilidade das características gerais, assim como da comparação.

O estudo é complementado pelo uso do Índice ISEI. Trata-se de uma escala criada através de uma ponderação das características socioeconômicas das pessoas inseridas em uma determinada ocupação; em geral, educação e renda. Apresenta natureza contínua e é utilizado ao longo do artigo como indicador-síntese da presença de uma dada estrutura ocupacional, além de nos auxiliar em diversos momentos ao confirmar a consistência das análises empreendidas. Essa medida de status foi desenvolvida por Ganzeboom, De Graaf e Treiman (1992) a partir dos códigos ocupacionais da International Standard Classification of Occupations - ISCO 88. Para aplicá-lo aos dados brasileiros, foi preciso converter os códigos ocupacionais do IBGE para ISCO 88. Ele mede os atributos da ocupação que converte a educação dos indivíduos em renda. Ao construir a escala, a ocupação foi hierarquizada de maneira a capturar a influência indireta da educação sobre a renda. Técnicas de optimal scaling foram usadas através de um algoritmo interativo envolvendo uma série de equações de regressão. A construção dessa escala base- 
ou-se no International Stratification and Mobility File, que consiste de um banco de dados com informações de diversos países, que vão desde países subdesenvolvidos como a Índia aos países mais desenvolvidos como os Estados Unidos. As variáveis utilizadas incluem educação, ocupação, idade e renda.

\section{ANALISANDO AS ESTRUTURAS SOCIAIS DAS DUAS CIDADES}

Iniciamos pela comparação das estruturas sociais medidas pelas classes EGP. Os dados sugerem uma situação muito mais similar entre as cidades do que se considera usualmente. A Tabela 1 expõe a presença relativa de indivíduos em cada classe, no conjunto de cada uma das duas cidades. Como se pode ver, a característica que mais chama a atenção é a grande similaridade das estruturas. Em ambas as cidades, as categorias mais presentes são Trabalhadores Não-Manuais de Rotina, seguidos dos Manuais Qualificados e Não-Qualificados. A categoria mais elevada, dos Profissionais e Administradores, envolve pouco mais de $15 \%$ do total, e a categoria Rural é praticamente inexistente. Embora talvez pudéssemos obter resultados diferentes se utilizássemos categorias mais abertas, o que indicaria a existência de diferenças nos detalhes das estruturas, a tabela nos informa que a conformação geral das duas estruturas é muito similar.

Tabela 1

Presença Relativa das Classes EGP no Rio de Janeiro e em São Paulo (2000)

(\%)

\begin{tabular}{l|c|c|c|c|c|c|c}
\hline Cidades & $\begin{array}{c}\text { I. Pro- } \\
\text { fissio- } \\
\text { nais e } \\
\text { Admi- } \\
\text { nistra- } \\
\text { dores }\end{array}$ & $\begin{array}{c}\text { II. Traba- } \\
\text { lhadores } \\
\text { Não-Ma- } \\
\text { nuais de } \\
\text { Rotina }\end{array}$ & $\begin{array}{c}\text { III. Pe- } \\
\text { quenos } \\
\text { Proprie- } \\
\text { tários }\end{array}$ & $\begin{array}{c}\text { IV. Traba- } \\
\text { lhadores } \\
\text { Manuais } \\
\text { Qualifica- } \\
\text { dos }\end{array}$ & $\begin{array}{c}\text { V. Traba- } \\
\text { lhadores } \\
\text { Manuais } \\
\text { Não-Qua- } \\
\text { lificados }\end{array}$ & VI. Rural & Total \\
\hline Rio de Janeiro & 16,2 & 30,1 & 4,3 & 25,5 & 23,2 & 0,6 & 100,0 \\
\hline São Paulo & 17,1 & 28,7 & 4,8 & 27,9 & 21,0 & 0,5 & 100,0 \\
\hline
\end{tabular}

Fontes: Cálculo próprio a partir de Scalon (2006) e Censo Demográfico 2000, IBGE.

Iniciamos o estudo por realizar uma análise de agrupamentos das áreas de ponderação segundo a presença proporcional das várias classes EGP. Foram executadas duas análises de cluster, utilizando a rotina K-means no software SPSS 13.0, tendo como unidades de análise as áreas de ponderação do Censo de 2000 (413 do Rio de Janeiro e 812 de 
São Paulo) e considerando a presença proporcional das várias classes EGP. Como a classe VI, de atividades rurais, não estava presente praticamente em nenhuma área, a análise a seguir inclui apenas as outras cinco classes EGP.

A aplicação do método em cada cidade indicou cinco agrupamentos como o número mais adequado para ambas as análises. A coesão das análises fica confirmada pelos valores médios do Índice ISEI em cada agrupamento em cada cidade. Os Boxplots a seguir apresentam a informação, sugerindo a robustez da análise, visto que o Índice ISEI não foi utilizado na construção dos agrupamentos.

Como podemos ver, os agrupamentos são bastante homogêneos. Os poucos outliers presentes em São Paulo contam com alguma presença da classe VI, atividades rurais, sugerindo conteúdos sociais distintos, mas muito específicos e localizados. Dado o pequeno número de áreas nessa condição, decidimos não torná-las discretas em um agrupamento separado.

\section{Gráfico 1}

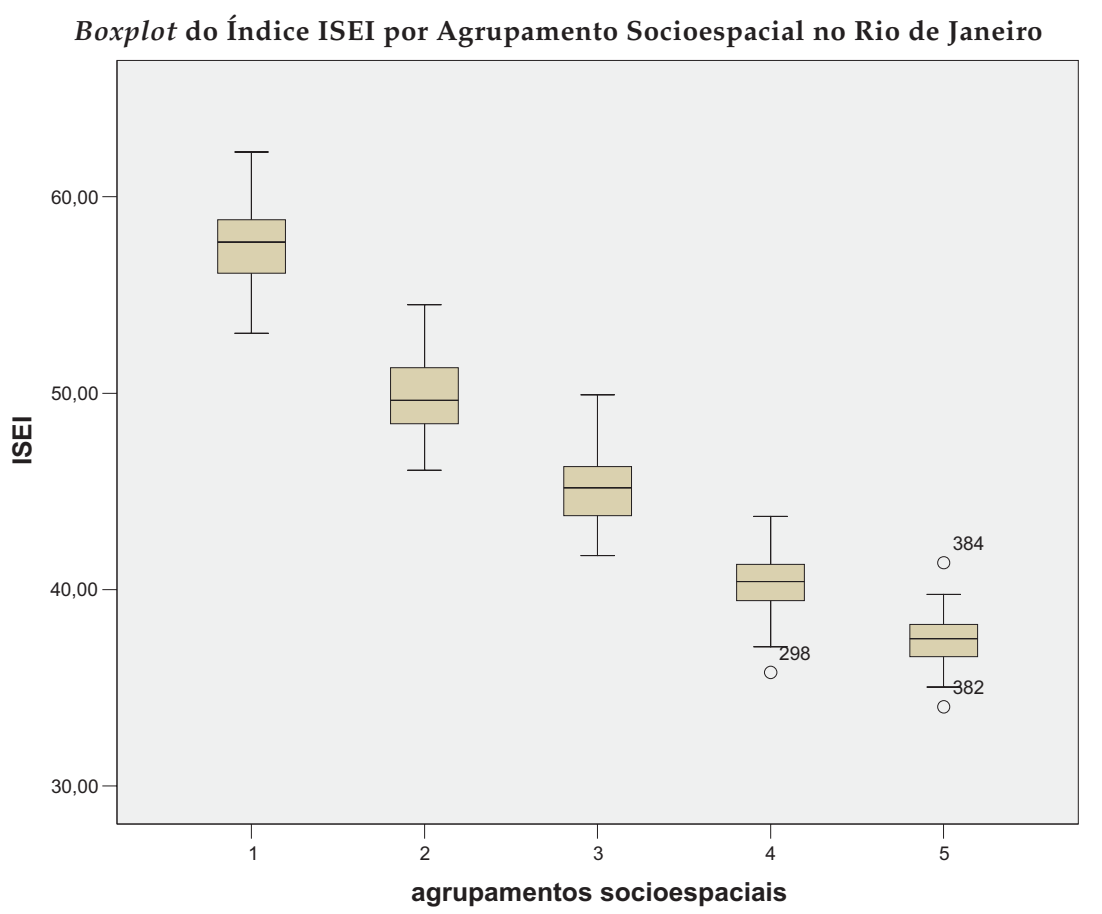

Fontes: Cálculo próprio a partir de Scalon (2006) e Censo demográfico 2000, IBGE. 


\section{Gráfico 2}

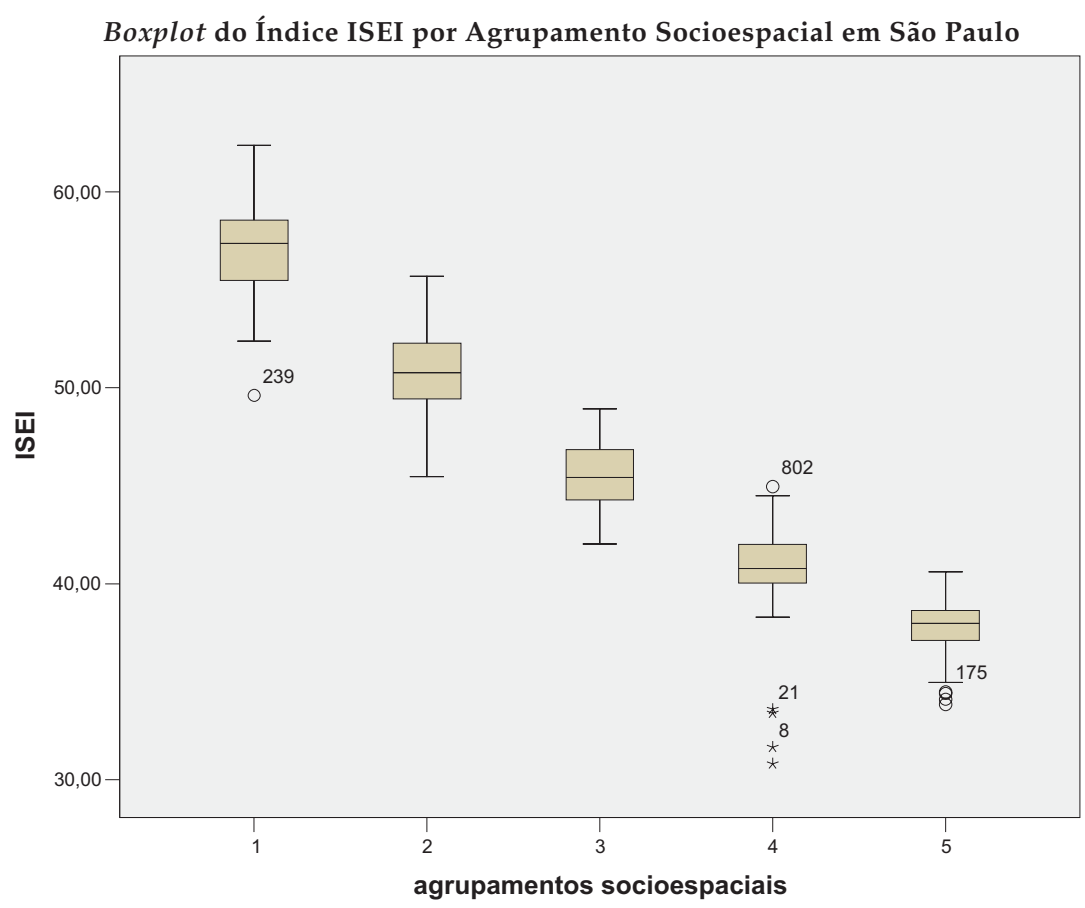

Fontes: Cálculo próprio a partir de Scalon (2006) e Censo demográfico 2000, IBGE.

A construção dos agrupamentos de forma separada para cada cidade poderia estar introduzindo algum viés na análise e tornando os resultados menos comparáveis. Por essa razão, decidimos repetir o procedimento para o conjunto das áreas de ponderação das duas cidades, aplicando-o a um banco de dados com 1.225 casos (413 do Rio de Janeiro e 812 de São Paulo). Vale dizer que os histogramas de cada classe em cada cidade são muito similares, resultando em distribuições conjuntas também muito parecidas. A análise de agrupamentos conjuntos com todos os casos também indicou cinco agrupamentos como a melhor solução, e o cruzamento do pertencimento aos agrupamentos gerados com dois bancos separados mostrou diferenças de classificação bastante pequenas entre agrupamentos ${ }^{3}$.

A robustez da análise foi novamente confirmada pela distribuição dos valores do Índice ISEI pelos agrupamentos. A comparação dos valores por agrupamento é feita no Gráfico 3. 


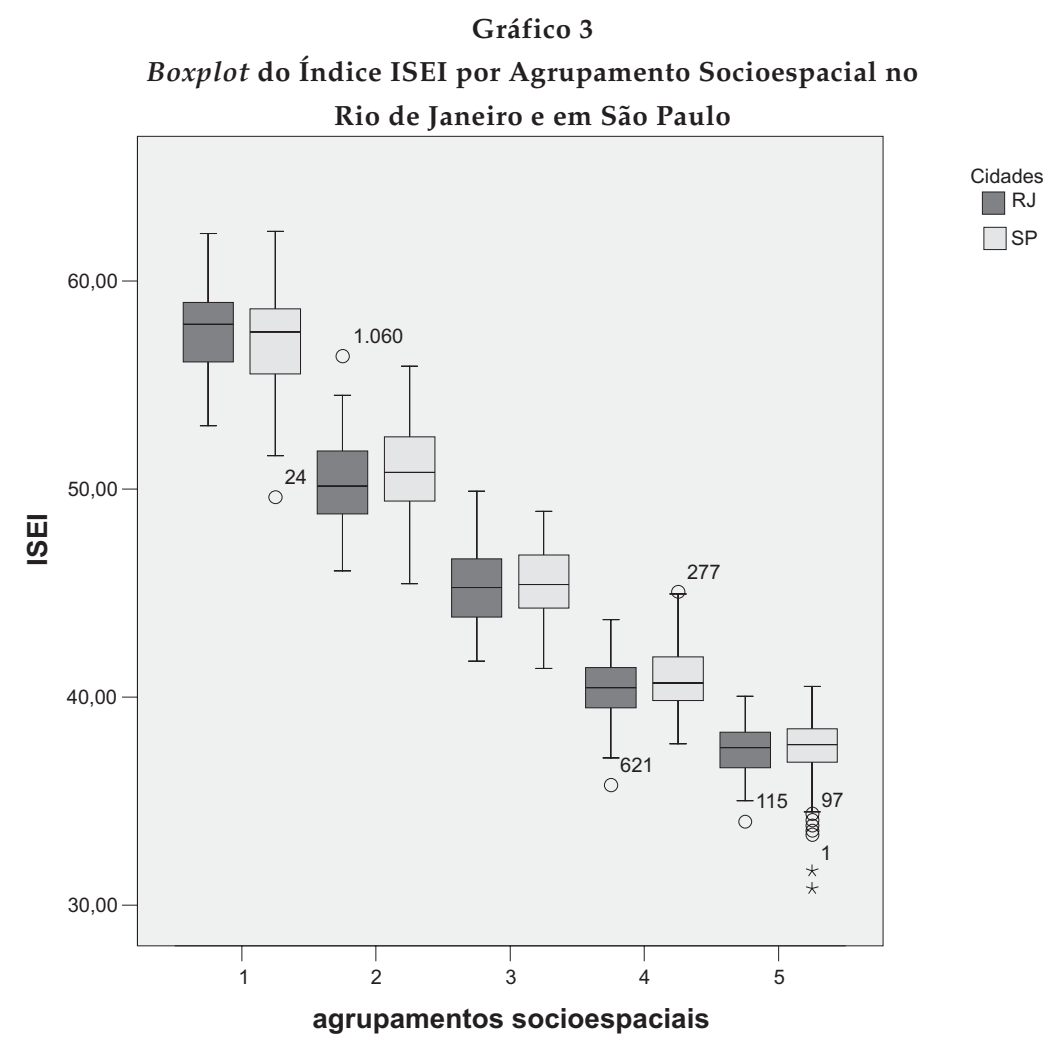

Fontes: Cálculo próprio a partir de Scalon (2006) e Censo Demográfico 2000, IBGE.

O gráfico confirma que os valores do índice por agrupamento em cada uma das cidades tendem a ser muito similares, assim como as respectivas distribuições. Para as cidades em seus conjuntos, obtemos um índice de 44,5 para São Paulo e de 43,3 para o Rio de Janeiro. Essa pequena diferença citada é significativa a $99 \%$ de confiança, mas distribui-se por diferenças ainda menos expressivas em todos os agrupamentos socioespaciais. Análise de variância indicou que apenas no caso do agrupamento 4 a diferença é significativa a 5\% (e favorável a São Paulo), sendo todas as demais diferenças entre médias não-significativas. As áreas com conteúdos rurais classificadas anteriormente como agrupamento 4 em São Paulo ficaram dessa vez no agrupamento 5, e representam os outliers do gráfico. De forma geral, podemos dizer que essas informações sugerem a presença de uma estrutura de ocupações muito similar, tanto para o conjunto da cidade quanto para os agrupamentos socioespaciais. 
Essa semelhança é novamente confirmada nas Tabelas 2 e 3, que apresentam as participações das classes EGP nos agrupamentos socioespaciais em cada cidade ${ }^{4}$. A Tabela 2 é organizada segundo a presença relativa, em cada agrupamento socioespacial, das classes EGP. A melhor forma de lê-la é comparando as linhas, que indicam os perfis sociais dos agrupamentos. Como podemos ver, os perfis são muito similares tanto para o conjunto das cidades quanto para os agrupamentos separadamente. Nos perfis das duas cidades, entre as pequenas diferenças existentes, vale reportar a presença um pouco maior da classe de Não-Manuais de Rotina (II) no Rio de Janeiro do que em São Paulo $(30,1 \%$ contra $28,7 \%)$ e uma proporção levemente mais elevada dos Trabalhadores Manuais Qualificados (IV) em São Paulo do que no Rio de Janeiro (27,9\% contra 25,6\%). Mesmo essas diferenças, entretanto, são muito pequenas. As diferenças entre as cidades nos agrupamentos, considerados separadamente, são ainda menores.

Tabela 2

Perfis dos Agrupamentos Socioespaciais Segundo Classes EGP, Rio de Janeiro e São Paulo (\%)

\begin{tabular}{|c|c|c|c|c|c|c|c|c|c|}
\hline & \multirow[b]{2}{*}{ 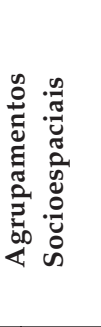 } & \multicolumn{7}{|c|}{ Classes EGP } & \multirow[b]{2}{*}{ 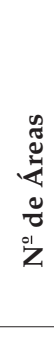 } \\
\hline & & 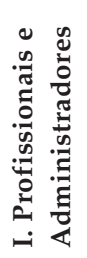 & 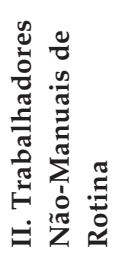 & 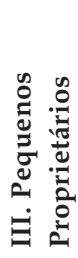 & 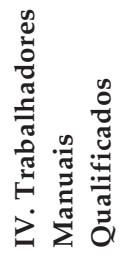 & 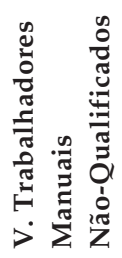 & 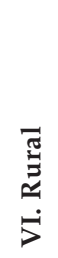 & 胥 & \\
\hline \multirow{6}{*}{ RJ } & 1 & 47,6 & 25,7 & 6,3 & 7,9 & 12,2 & 0,3 & 100,0 & 30 \\
\hline & 2 & 27,2 & 34,7 & 5,2 & 17,9 & 14,8 & 0,2 & 100,0 & 42 \\
\hline & 3 & 14,9 & 35,7 & 4,2 & 25,6 & 19,3 & 0,3 & 100,0 & 109 \\
\hline & 4 & 7,8 & 28,7 & 3,8 & 31,3 & 27,8 & 0,7 & 100,0 & 130 \\
\hline & 5 & 5,2 & 22,2 & 3,6 & 32,0 & 35,3 & 1,7 & 100,0 & 102 \\
\hline & Total & 16,2 & 30,1 & 4,3 & 25,6 & 23,2 & 0,6 & 100,0 & 413 \\
\hline \multirow{6}{*}{ SP } & 1 & 46,3 & 25,6 & 8,3 & 8,3 & 11,1 & 0,2 & 100,0 & 73 \\
\hline & 2 & 28,1 & 34,0 & 7,0 & 18,4 & 12,4 & 0,2 & 100,0 & 132 \\
\hline & 3 & 16,3 & 33,7 & 4,8 & 27,6 & 17,5 & 0,2 & 100,0 & 209 \\
\hline & 4 & 9,5 & 27,4 & 3,4 & 34,2 & 25,1 & 0,5 & 100,0 & 244 \\
\hline & 5 & 5,8 & 20,2 & 3,1 & 36,4 & 32,8 & 1,7 & 100,0 & 154 \\
\hline & Total & 17,1 & 28,7 & 4,8 & 27,9 & 21,0 & 0,5 & 100,0 & 812 \\
\hline
\end{tabular}

Fontes: Cálculo próprio a partir de Scalon (2006) e Censo demográfico 2000, IBGE. 
A Tabela 3 complementa a informação apresentando a distribuição relativa de cada classe EGP nos agrupamentos socioespaciais e pode ser lida como indicando a distribuição (ou concentração) das classes segundo agrupamentos. Embora as semelhanças persistam, nesse caso algumas pequenas diferenças devem ser notadas. Para o conjunto da cidade, os agrupamentos 2 e 4 tendem a ser mais presentes populacionalmente em São Paulo do que no Rio de Janeiro, ao contrário dos agrupamentos 3 e 1 , proporcionalmente mais elevados no caso carioca, sendo que apenas para os agrupamentos 2 e 3 as diferenças são de certa monta. As áreas do agrupamento 2 em São Paulo alojam proporções mais elevadas das classes de I a IV quando comparadas com o Rio de Janeiro, mas com diferenças substantivas nas classes mais bem posicionadas na estrutura social - classes I, II e III, sugerindo que as áreas desse agrupamento alojam população levemente mais bem posicionada em São Paulo. Por outro lado, o agrupamento 3 apresenta a participa-

Tabela 3

Distribuição das Classes EGP nos Agrupamentos Socioespaciais, Rio de Janeiro e São Paulo (\%)

\begin{tabular}{|c|c|c|c|c|c|c|c|c|c|}
\hline \multirow{2}{*}{\multicolumn{2}{|c|}{ 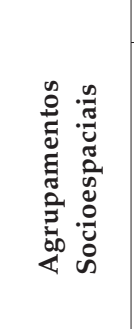 }} & \multicolumn{7}{|c|}{ Classes EGP } & \multirow[b]{2}{*}{ 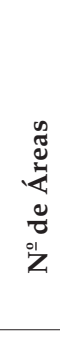 } \\
\hline & & 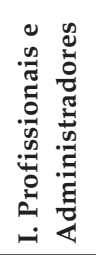 & 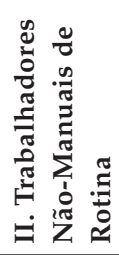 & 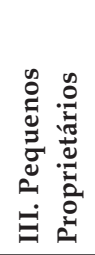 & 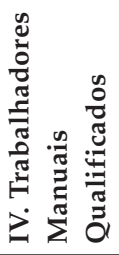 & 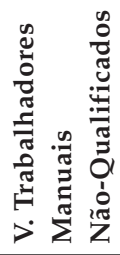 & 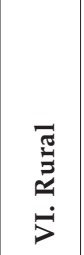 & 胥 & \\
\hline \multirow[t]{6}{*}{ RJ } & 1 & 31,1 & 9,0 & 15,4 & 3,3 & 5,6 & 5,4 & 10,6 & 30 \\
\hline & 2 & 22,8 & 15,7 & 16,3 & 9,5 & 8,7 & 5,2 & 13,6 & 42 \\
\hline & 3 & 26,3 & 34,0 & 27,8 & 28,8 & 23,8 & 13,2 & 28,7 & 109 \\
\hline & 4 & 14,2 & 28,4 & 26,0 & 36,5 & 35,5 & 30,6 & 29,7 & 130 \\
\hline & 5 & 5,5 & 12,8 & 14,6 & 21,9 & 26,5 & 45,6 & 17,4 & 102 \\
\hline & Total & 100,0 & 100,0 & 100,0 & 100,0 & 100,0 & 100,0 & 100,0 & 413 \\
\hline & & & & & & & & & \\
\hline \multirow[t]{6}{*}{ SP } & 1 & 25,4 & 8,4 & 16,4 & 2,8 & 4,9 & 4,2 & 9,4 & 73 \\
\hline & 2 & 26,6 & 19,2 & 23,7 & 10,7 & 9,5 & 5,2 & 16,2 & 132 \\
\hline & 3 & 24,8 & 30,6 & 26,2 & 25,8 & 21,6 & 9,8 & 26,1 & 209 \\
\hline & 4 & 17,3 & 29,7 & 22,4 & 38,1 & 37,0 & 26,4 & 31,1 & 244 \\
\hline & 5 & 5,8 & 12,1 & 11,3 & 22,6 & 26,9 & 54,4 & 17,3 & 154 \\
\hline & Total & 100,0 & 100,0 & 100,0 & 100,0 & 100,0 & 100,0 & 100,0 & 812 \\
\hline
\end{tabular}

Fontes: Cálculo próprio a partir de Scalon (2006) e Censo demográfico 2000, IBGE. 
ção mais elevada de todas as classes no Rio de Janeiro, embora as maiores diferenças entre as duas cidades se localizem nas classes II, IV e VI. Observando os perfis das classes individualmente, devemos destacar que os profissionais mais bem posicionados (classes I e II) tendem a se concentrar mais no agrupamento mais alto (1) no Rio de Janeiro do que em São Paulo. Nesta cidade, eles tendem a se espalhar mais, estando mais presentes inclusive nos agrupamentos 2 e 3 . Voltamos a destacar, entretanto, que essas diferenças são muito pequenas em termos relativos. A concentração das classes pior inseridas na estrutura, por outro lado, é praticamente idêntica nas duas cidades.

Em seu conjunto, as informações sugerem a existência de espaços (agrupamentos de áreas) bastante similares nas duas cidades. Isso indica que não apenas as estruturas sociais são similares, mas também que a distribuição dos indivíduos no interior das áreas de ponderação é bastante assemelhada, embora os agrupamentos mais bem posicionados se encontrem levemente mais segregados no Rio de Janeiro, considerando essa escala de análise. Como as áreas de ponderação representam unidades bastante desagregadas, essa semelhança sugere padrões similares de microssegregação, visto que aponta para a concentração de classes em vizinhanças de pequena escala. Assim, não apenas a estrutura geral da distribuição dos agrupamentos, mas também a segregação social no espaço, é muito similar, embora os profissionais de melhor posição estejam levemente mais concentrados em seus espaços no Rio de Janeiro do que em São Paulo.

Tentando analisar conjuntamente as informações discutidas anteriormente, podemos ainda sugerir a seguinte caracterização dos agrupamentos:

- Agrupamento 1: É ocupado predominantemente por Profissionais / Administradores e Trabalhadores Não-Manuais de Rotina (mais de $72 \%$ nas duas cidades). Nesse agrupamento, a classe I apresenta sua maior concentração no Rio de Janeiro e a segunda maior em São Paulo.

- Agrupamento 2: Similar ao anterior, embora em patamar mais baixo (62\% das classes citadas no Agrupamento 1) e tendo nesse caso a classe predominante como os Profissionais Não-Manuais de Rotina - II (34\%). Além disso, nesse agrupamento, a presença dos Manuais Qualificados e Não-Qualificados (das classes IV e V) é expressiva (mais de $30 \%$ contra $20 \%$ no anterior). Esse agrupamento e o anterior podem ser considerados como os espaços dos profissionais e trabalhadores não-manuais. 
- Agrupamento 3: A predominância da classe II mantém-se no mesmo patamar (35\%), e a classe dos profissionais tende a cair ainda mais, sendo ultrapassada pelos Manuais Qualificados e Não-Qualificados (IV e V), com cerca de $45 \%$. A classe que mais concentra sua participação é a dos Não-Manuais de Rotina (II). É o agrupamento com perfis mais próximos do perfil médio de cada cidade e inclui certamente os espaços mais heterogêneos socialmente das cidades.

- Agrupamento 4: As classes predominantes são a IV, II e V, sendo que os Manuais Qualificados superam os 30\%, e os trabalhadores manuais como um todo alcançam $60 \%$. Esse agrupamento e o que segue podem ser considerados sinteticamente como espaços predominantemente dos trabalhadores manuais.

- Agrupamento 5: As classes manuais chegam a quase $70 \%$, e a de profissionais fica em torno de $5 \%$, invertendo o perfil do espaço dos profissionais (Agrupamento 1). No caso do Rio de Janeiro, a classe dos Trabalhadores Manuais Não-Qualificados chega mesmo a predominar sobre a dos Manuais Qualificados, o que não acontece em São Paulo.

Avançaremos na próxima seção na análise da distribuição espacial e da segregação das classes, mas a caracterização dos agrupamentos de unidades espaciais ainda nos auxilia a compreender algumas de suas facetas. Embora haja estruturas claras tanto de concentração quanto de predominância das classes nos agrupamentos (e conseqüentemente no espaço), metrópoles como o Rio de Janeiro e São Paulo são marcadas pela heterogeneidade e dispersão dos agrupamentos. Observemos apenas alguns exemplos retirados da tabela anterior. Embora a classe dos profissionais seja predominante no Agrupamento 1, apenas algo entre $25 \%$ e $30 \%$ da população dessa classe reside em áreas desse tipo. Olhando para o outro extremo da distribuição, embora as classes manuais sejam amplamente predominantes nos agrupamentos 4 e 5 , cerca de $40 \%$ da população dessas classes reside em áreas de outro tipo. Se observarmos apenas o Agrupamento 5, que inclui cerca de $17 \%$ da população de cada cidade, encontraremos apenas entre 20\% e 25\% dos indivíduos classificados nas classes manuais. Resultados desse tipo aparecem em outras cidades, como Paris, Madri e Atenas (Preteceille, 2006; Leal Maldonado, 2007 e Maloutas, 2007). Quando falamos de alta segregação, portanto, chamamos a atenção para um fenômeno importante com inúmeras conseqüências, mas que de forma alguma é o único processo em curso. 


\section{ANALISANDO A DISTRIBUIÇÃO ESPACIAL DAS CATEGORIAS}

Nesta seção, analisamos as informações relativas às classes EGP e ao Índice ISEI, investigando seus padrões espaciais e a questão da segregação urbana na escala da cidade como um todo e entre áreas de ponderação. Para iniciar a análise, os Mapas 1 e 2 apresentam a distribuição do ISEI para as duas cidades. Como se pode ver, a distribuição do índice é relativamente radial, concêntrica e muito segregada, embora com heterogeneidades localizadas significativas. Na verdade, essa heterogeneidade tende a ser maior nas áreas intermediárias e no início das áreas periféricas. Resultados similares foram encontrados para Rio

Mapas 1 e 2

Distribuição do ISEI nas Áreas de Ponderação, Rio de Janeiro e São Paulo Índice Socioeconômico (Média)
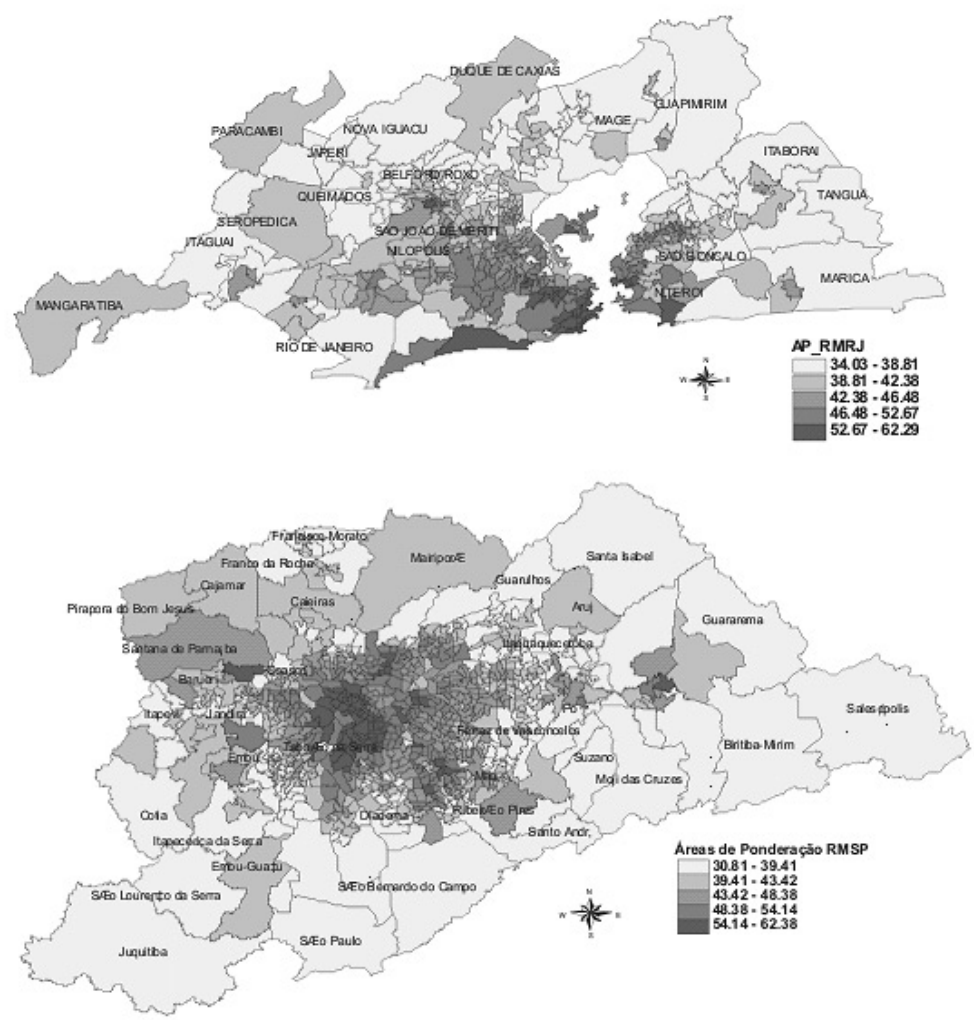

Fontes: Elaboração própria a partir de Scalon (2006), IBGE e cartografias do Centro de Estudos da Metrópole/Cebrap. 
de Janeiro, Belo Horizonte e Salvador, embora com metodologia e categorias distintas (Ribeiro e Lago, 2000; Mendonça, 2003; Carvalho, Souza e Pereira, 2004).

A análise dos padrões espaciais visíveis nos mapas anteriores fica mais precisa se lançarmos mão de técnicas de mensuração da concentração dos agrupamentos sociais ou da segregação social no espaço. Essas técnicas usualmente avaliam o grau de associação de um dado indicador para uma área específica, com os relativos às áreas vizinhas, investigando a existência de padrões aleatórios ou de correlações espaciais. Neste artigo, utilizaremos os Índices de Moran - Global e Local. O chamado Índice de Moran Global avalia o grau de associação entre uma dada variável em uma área e o valor dessa área para o conjunto das áreas, conforme a expressão abaixo:

$$
\mathrm{I}=\frac{\sum_{i=1}^{n} \sum_{j=1}^{n} w_{i j}\left(z_{i}-\bar{z}\right)\left(z_{j}-\bar{z}\right)}{\sum_{i=1}^{n}\left(z_{i}-z\right)^{2}}
$$

Na equação acima, $n$ é o número de áreas, $z_{\mathrm{i}}$ o valor do atributo considerado na área $i, z$ é o valor médio do atributo na região de estudo e $w_{\mathrm{ij}}$ os elementos da matriz normalizada de proximidade espacial. Nesse caso, a correlação será computada apenas para os vizinhos de primeira ordem no espaço, conforme estabelecido pelos pesos $w_{\mathrm{ij}}$.

O índice varia entre -1 e +1 , sendo que valores negativos significam correlações espaciais negativas, e valores positivos indicam correlações espaciais positivas. Como o Índice de Moran Global representa uma síntese das associações das várias localizações, não sabemos por ele onde as concentrações apontadas se verificam. Apesar disso, esse índice representa uma ferramenta importante para comparações amplas. A Tabela 4 apresenta o Índice para o conjunto das duas cidades.

O conjunto dos índices sugere padrões de segregação muito similares nas duas cidades. Nas classes I e IV, São Paulo apresenta índices levemente superiores, e nas classes II e V os índices cariocas são levemente mais altos. Isso é interessante, pois vimos na Tabela 3 que, em São Paulo, a concentração dos indivíduos pertencentes à classe I em espaços em que eles eram predominantes era menor do que no Rio de Janeiro. A diferença deve-se a duas dimensões. Em primeiro lugar, o Índice de Moran Global aponta para a concentração, mas não especifica onde 
Eduardo Marques, Celi Scalon e Maria Aparecida Oliveira

Tabela 4

Índice de Moran Global, Rio de Janeiro e São Paulo

\begin{tabular}{lcc}
\hline \multirow{2}{*}{ Classes EGP } & \multicolumn{2}{c}{ Índice de Moran } \\
\cline { 2 - 3 } & RMRJ $^{*}$ & RMSP** $^{*}$ \\
\hline I. Profissionais e Administradores & 0,74 & 0,78 \\
II. Não-Manual de Rotina & 0,65 & 0,62 \\
III. Pequenos Proprietários & 0,18 & 0,54 \\
IV. Manual Qualificado e Supervisores & 0,72 & 0,78 \\
V. Manual Não-Qualificado & 0,64 & 0,62 \\
VI. Rural & 0,50 & 0,51 \\
Índice ISEI & 0,69 & 0,73 \\
\hline
\end{tabular}

Fontes: Scalon (2006) a partir de Censo Demográfico 2000, IBGE.

* Região Metropolitana do Rio de Janeiro.

** Região Metropolitana de São Paulo.

esta ocorre. Além disso, entretanto, a informação da Tabela 3 diz respeito à maior ou menor concentração dos indivíduos nas áreas de ponderação de determinado tipo, indicando maior ou menor contigüidade social entre indivíduos na escala micro. O Índice de Moran, diferentemente, descreve a situação de contigüidade espacial entre áreas de ponderação. Apenas no caso da classe III, a diferença entre os índices é elevada, mas trata-se de uma classe muito pouco freqüente e, portanto, o resultado pode-se dever ao pequeno número de casos. De qualquer forma, não poderíamos esperar uma concentração geográfica dessa classe, porque ela é bastante heterogênea. A segregação do Índice ISEI também é muito similar, embora mais elevada em São Paulo, que, como já vimos, também apresenta um índice médio um pouco mais alto.

Além da similaridade dos índices de Moran nas duas cidades, entretanto, vale destacar que nas duas cidades a classe I apresenta os índices mais elevados de segregação, confirmando resultados de trabalhos sobre outras cidades que indicam que a estrutura da segregação é impulsionada pela segregação dos agrupamentos mais bem posicionados socialmente.

Além dessa medida, utilizamos o Índice de Moran Local - Lisamap, ou "Local indicators of spatial association" (Anselin, 1995). As técnicas de análise de associação espacial local comparam os valores de uma variável em uma dada área de ponderação com os valores encontrados em seus vizinhos, permitindo testar a hipótese de aleatoriedade espacial. Em outras palavras, a medida fornece medidas para o estudo de pa- 
drões locais, apropriadas para análises intra-urbanas. Desse modo, permite a identificação de agrupamentos espaciais com base nos valores das variáveis analisadas.

Para calcular o Moran Local, é necessário construir uma matriz de vizinhança ou proximidade espacial. Inicialmente, testamos uma matriz de primeira e outra de segunda ordem. Como os resultados foram semelhantes, trabalhamos sempre com uma matriz de primeira ordem, baseada nos quatro vizinhos mais próximos. O Índice de Moran Localé obtido a partir da equação:

$\mathrm{I}_{i} \frac{\sum_{j=1}^{n} w_{i j} z_{j}}{\sum_{j=1}^{n} z_{j}^{2}}$, onde $w_{i j}$ é um elemento da matriz de vizinhança espacial $w$, que indica as áreas $i$ e $j$, e $n$ é o número de áreas.

Iniciamos pelo cálculo do Índice de Moran Local para a medida ISEI de status socioeconômico ocupacional. Os Mapas 3 e 4 a seguir apresentam a informação para as duas cidades. Eles indicaram para o ISEI uma forte tendência de concentração espacial das melhores condições sociais, com os valores mais baixos dos índices localizados perifericamente.

$$
\text { Mapa } 3
$$

Lisa de ISEI, Rio de Janeiro

Índice Socioeconômico - RMRJ 2000

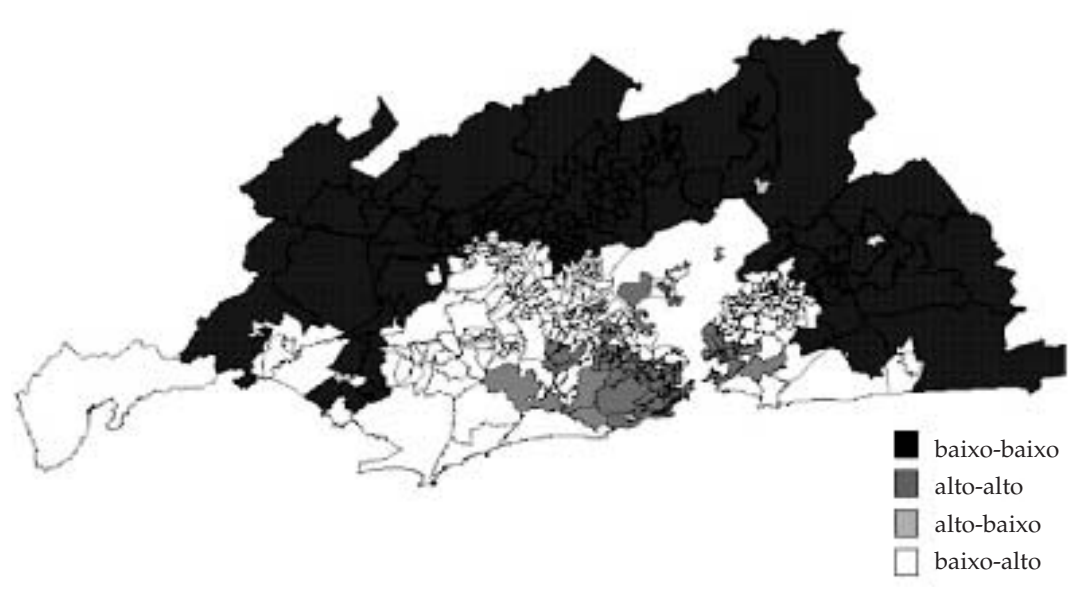




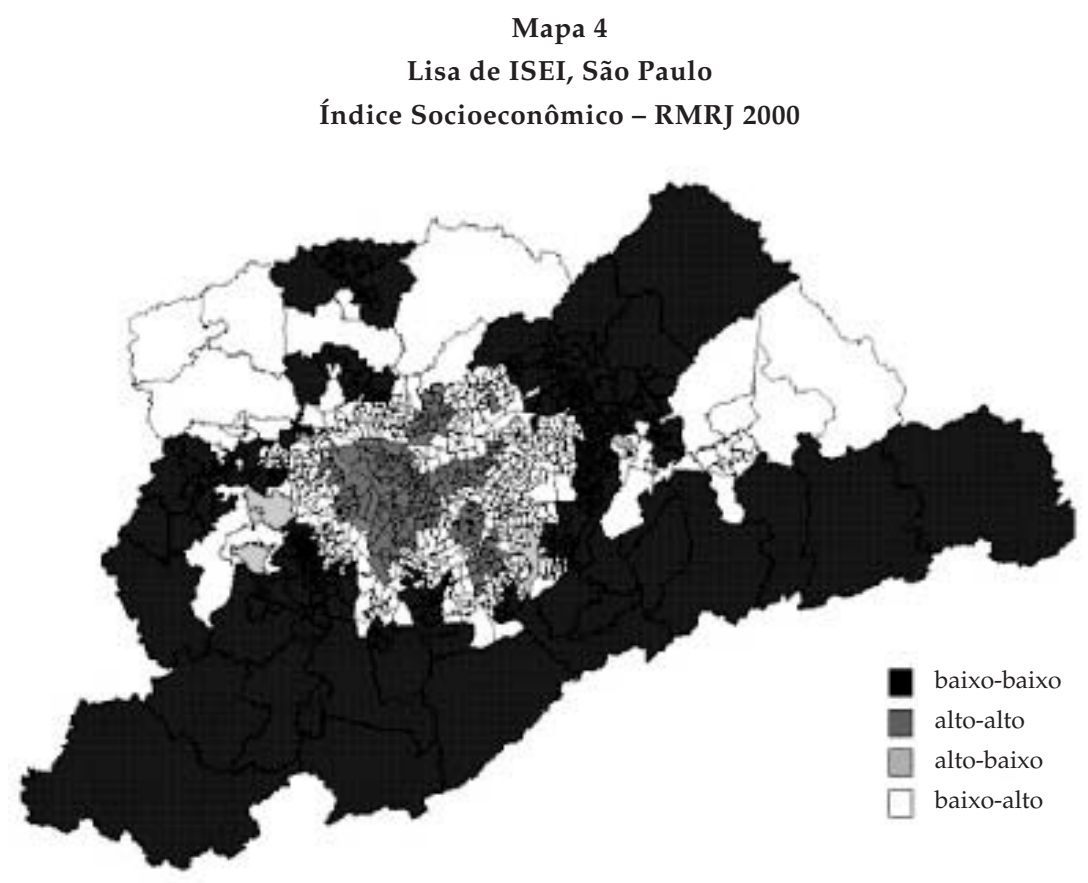

Fontes: Elaboração própria a partir de Scalon (2006), IBGE e cartografias do Centro de Estudos da Metrópole/Cebrap.

Poderíamos analisar em Lisamaps a distribuição das diversas classes, mas uma forma de observarmos o fenômeno de forma mais sucinta diz respeito à construção de gráficos de distribuição das classes EGP segundo as quatro condições de correlação entre vizinhos do Lisa elaborado com os valores do ISEI. Os Gráficos 4 e 5 apresentam a informação. Como podemos ver, os formatos dos gráficos são bastante similares, com leve diferença no comportamento das classes Manual Não-Qualificado e Manual Qualificado e Supervisores. A presença dessas classes é levemente superior em áreas baixo-baixo em São Paulo, que correspondem às periferias. A sua presença em áreas alto-baixo, em compensação, é menor, sugerindo uma estrutura urbana mais homogênea, tanto nas áreas mais periféricas (homogêneas nas classes menos qualificadas), quanto em áreas de índice elevado cercadas de áreas com índice baixo (homogêneas nas classes mais qualificadas). Por outro lado, o Rio de Janeiro apresenta uma presença relativa mais elevada das classes de Profissionais e Não-Manuais de Rotina (I e II) em áreas alto-alto, sugerindo uma maior concentração desses agrupamentos nos espaços mais elitizados, como já tínhamos visto na Tabela 3. 
Gráfico 4

Distribuição das Classes por Agrupamento, Rio de Janeiro

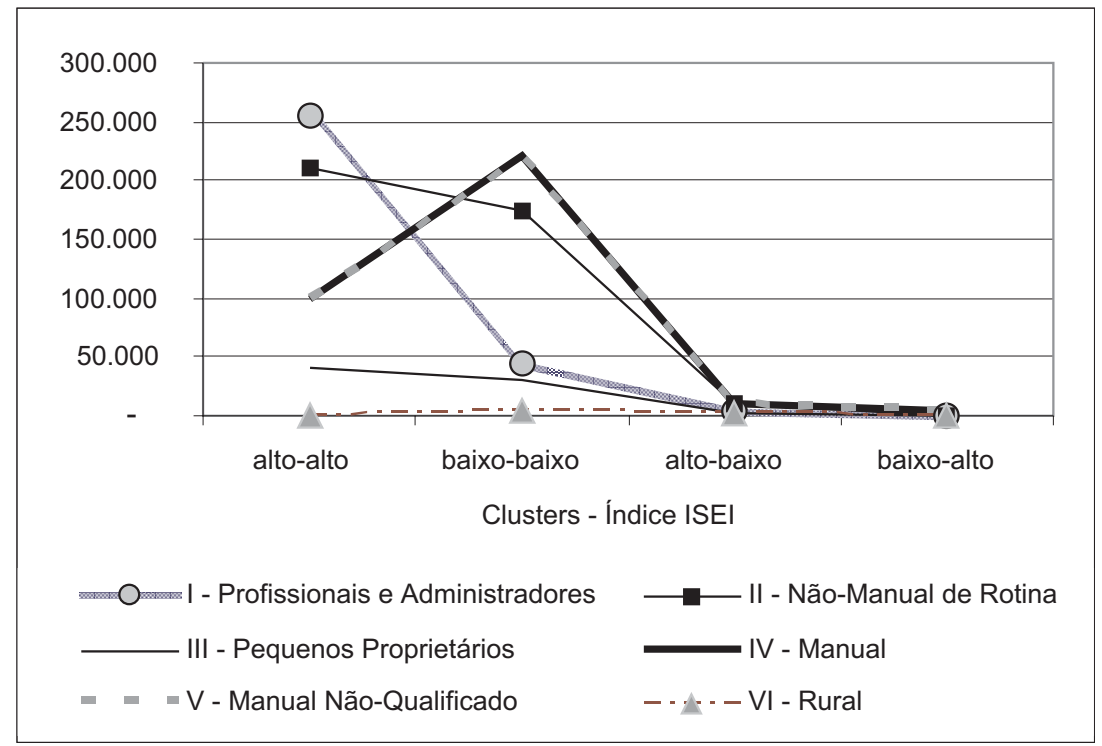

Fontes: Cálculo próprio a partir de Scalon (2006), IBGE e cartografias do Centro de Estudos da Metrópole/Cebrap.

Gráfico 5

Distribuição das Classes por Agrupamento, São Paulo

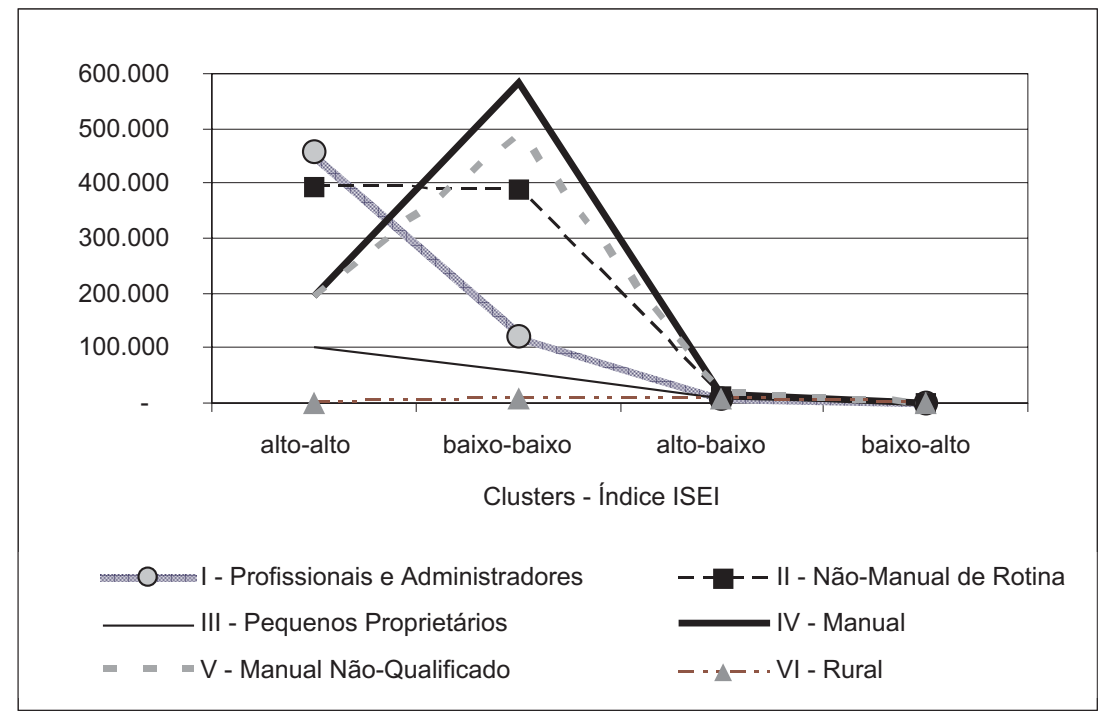

Fontes: Cálculo próprio a partir de Scalon (2006), IBGE e cartografias do Centro de Estudos da Metrópole/Cebrap. 
Resumindo, podemos dizer que todos os índices obtidos e os mapas com os níveis de associação espacial local confirmam as informações da primeira parte da análise, apontando para dois resultados gerais. $\mathrm{O}$ primeiro está associado às amplas evidências de similaridade entre as duas cidades, tanto com relação à presença relativa das classes quanto à sua distribuição espacial. Por outro lado, os dados também indicam a presença de intensa segregação espacial entre as classes em ambas as cidades, com características levemente mais acentuadas na RMSP.

\section{À GUISA DE CONCLUSÃO}

Ao longo deste artigo, exploramos as estruturas sociais do Rio de Janeiro e de São Paulo a partir de dados das classes EGP e do Índice ISEI. Como vimos, os resultados apontam para a existência de grande similaridade entre as duas cidades, tanto em termos da estrutura encontrada quanto em relação aos padrões de segregação social no espaço do conjunto de cada metrópole.

Essa similaridade aparece em primeiro lugar na simples comparação da distribuição dos indivíduos pelas categorias EGP. O mesmo tipo de resultado foi encontrado quando realizamos a análise de agrupamentos das áreas de ponderação pela presença relativa das categorias. Os agrupamentos encontrados nas duas cidades são muito parecidos, tanto pela sua composição relativa quanto pelos seus índices socioeconômicos médios medidos pelo ISEI. Isso indica que não apenas as estruturas sociais são similares, mas também que a distribuição dos indivíduos no interior das áreas de ponderação é bastante assemelhada. Como as áreas de ponderação representam unidades bastante desagregadas, essa semelhança pode ser considerada como indicador da presença de padrões de microssegregação bastante parecidos. A única diferença a considerar diz respeito a uma concentração um pouco maior no Rio de Janeiro do que em São Paulo dos indivíduos mais bem posicionados na estrutura social em áreas de ponderação classificadas como melhores. Entretanto, essas informações nada nos informam sobre os padrões de contigüidade em escalas maiores.

Realizamos então uma análise sobre a distribuição espacial das categorias EGP. Como vimos, os Índices de Moran indicam uma estrutura espacial altamente segregada (e semelhante) nas duas cidades, tanto sob o ponto de vista das classes EGP quanto do ISEI. Os Mapas de Moran Local do ISEI confirmam essa segregação em larga escala, assim como 
os gráficos da presença relativa das classes nas diversas condições de contigüidade do Lisa. Apesar das semelhanças nos padrões gerais, uma exceção no caso do Rio de Janeiro está nas favelas, discriminadas pela análise como áreas de baixo-alto, provavelmente inseridas na região mais elitizada da cidade, assim como na região suburbana. Por outro lado, os resultados apontam para a existência de maior heterogeneidade nas áreas de piores condições sociais em São Paulo, localizadas segundo os mapas nas periferias, o que confirma resultados anteriores de estudos sobre São Paulo.

(Recebido para publicação em agosto de 2007) (Versão definitiva em outubro de 2007)

\section{NOTAS}

1. ISEI é a abreviatura de International Social-Economic Index of Occupational Status, nomenclatura utilizada na literatura. Ver Ganzeboom, De Graaf e Treiman (1992).

2. Ver os depoimentos publicados sobre o uso da categoria na revista Espaço e Debates, no $-42,2002$.

3. Da classificação final, apenas nove áreas no Rio de Janeiro (3,6\%) e 56 em São Paulo $(6,9 \%)$ foram reclassificadas dos agrupamentos originais de cada cidade, e todas as diferenças de classificações pertenciam a agrupamentos originais contíguos.

4. Vale observar que essa semelhança confirma resultados de trabalhos anteriores, utilizando categorias socioocupacionais francesas mais detalhadas para 1991 como Ribeiro e Lago (2000). 


\section{REFERÊNCIAS BIBLIOGRÁFICAS}

ANSELIN, L. (1995), “Local Indicator of Spatial Association - LISA”. Geografical Analysis, no 27, pp. 91-115.

BICHIR, R. (2006), Segregação e Acesso a Políticas Públicas no Município de São Paulo. Dissertação de mestrado em Ciência Política, Faculdade de Filosofia, Letras e Ciências Humanas/USP, São Paulo.

BONDUKI, N. e ROLNIK, R. (1982), “Periferia da Grande São Paulo: Reprodução do Espaço como Expediente de Reprodução da Força de Trabalho", in E. Maricato (org.), A Produção Capitalista da Casa (e da Cidade) do Brasil Industrial. São Paulo, Alfa-Ômega.

BOURDIEU, P. (1989), O Poder Simbólico. Rio de Janeiro, Bertrand.

BRASILEIRO, A. (org.). (1976), Região Metropolitana do Rio de Janeiro: Serviços de Interesse Comum. Rio de Janeiro, Ibam.

CAMARGO, C. (org.). (1976), São Paulo, 1975 - Crescimento e Pobreza. São Paulo, Ed. Loyola.

CARVALHO, I., SOUZA, Â. e PEREIRA, G. (2004), “Polarização e Segregação Socioespacial em uma Metrópole Periférica". Cadernos CRH, vol. 17, no 41, pp. 281-297.

CENTRO DE ESTUdOS DA METRÓPOLE - CEM. (2004), Mapa da Vulnerabilidade Social da População da Cidade de São Paulo. CEM/Pmsp/Sesc.

ERIKSON, R. GOLDTHORPE, J. H. e PORTOCARRERO, L. (1979), “Intergenerational Class Mobility in Three Western European Societies". British Journal of Sociology, vol. 30, pp. 415-441.

GANZEBOOM, H. B. G., DE GRAAF, P. M. e TREIMAN, D. J. (1992), “A Standard International Socio-Economic Index of Occupational Status". Social Science Research, no 21, pp. 1-56.

KOWARICK, L. (1979), A Espoliação Urbana. Rio de Janeiro, Paz e Terra.

(2000), Escritos Urbanos. São Paulo, Editora 34.

LAGO, L. (2002), “A Lógica Segregadora na Metrópole Brasileira: Novas Teses sobre Antigos Processos". Cadernos IPPUR, Ano XV/XVI, número especial.

LEAL MALDONADO, J. (2007), “Desigualdad Social, Segregación y Mercado de Vivienda en Madrid", in J. Leal Maldonado (org.), Vivienda y Segregación en las Grandes Ciudades Europeas. Madrid, Universidad Computense de Madrid.

MALOUTAS, T. (2007), “Segregación, Polarización Social y Desigualdad en Atenas durante los 90", in J. Leal Maldonado (org.), Vivienda y Segregación en las Grandes Ciudades Europeas. Madrid, Universidad Computense de Madrid.

MARICATO, E. (1977), A Proletarização do Espaço sob a Grande Indústria. O Caso de São Bernardo do Campo. Tese de doutorado, FAU/USP, São Paulo.

MARQUES, E. e BITAR, S. (2002), “Espaço e Agrupamentos Sociais na Metrópole Paulistana". Novos Estudos Cebrap, no 64, pp. 123-131.

MARQUES, E. e TORRES, H. (2005), São Paulo: Segregação, Pobreza Urbana e Desigualdade Social. São Paulo, Senac. 
MENDONÇA, J. (2003), "Belo Horizonte: A Metrópole Segregada", in J. Mendonça e M. Godinho (orgs.), População, Espaço e Gestão na Metrópole: Novas Configurações, Velhas Desigualdades. Belo Horizonte, PUC-Minas.

PRETECEILLE, E. (2006). “La Ségrégation Sociale a-t-elle Augmenté? La Métropole Parisiense entre Polarisation et Mixité". Societé Contemporaines, no 62.

RIBEIRO, L. (2002), “Segregação, Acumulação Urbana e Poder: Classes e Desigualdades na Metrópole do Rio de Janeiro". Cadernos IPPUR, Ano XV /XVI, número especial, pp. 79-103.

__ e PRETECEILLE, E. (1999), “Tendências da Segregação Social em Metrópoles Globais e Desiguais: Paris e Rio de Janeiro nos Anos 80". Revista Brasileira de Ciências Sociais, vol. 14, no 40, pp. 143-162.

RIBEIRO, L. e LAGO, L. (2000), “O Espaço Social das Grandes Metrópoles Brasileiras”. Revista Brasileira de Estudos Urbanos e Regionais, vol. 2, no 3, pp. 111-129.

SANTOS, C. e BRONSTEIN, O. (1978), "Meta-Urbanização - O Caso do Rio de Janeiro". Revista de Administração Municipal, vol. 25, no 149.

SCALON, C. (1999), Mobilidade Social no Brasil: Padrões e Tendências. Rio de Janeiro, Revan.

. (2006), Relatório Final de Bolsa de Professora Visitante. São Paulo, CEM/FAPESP.

SPOSATI, A. (coord.). (1996), Mapa da Exclusão/Inclusão da Cidade de São Paulo. São Paulo, EDUC.

TASCHNER, S. e BÓGUS, L. (1999), “São Paulo como Patchwork: Unindo Fragmentos de uma Cidade Segregada". Cadernos Metrópole, no 1, pp. 43-98.

. (2000), "A Cidade dos Anéis: São Paulo", in L. C. Queiroz (org.), O Futuro das Metrópoles: Desigualdades e Governabilidade. Rio de Janeiro, Revan.

VALLADARES, L. e PRETECEILLE, E. (2000), “Favela, Favelas: Unidade ou Diversidade da Favela Carioca", in L. C. Ribeiro (org.), O Futuro das Metrópoles: Desigualdades e Governabilidade. Rio de Janeiro, Revan.

VETTER, D. (1981), “A Segregação Residencial da População Economicamente Ativa na Região Metropolitana do Rio de Janeiro, Segundo Agrupamentos de Rendimento Mensal". Revista Brasileira de Geografia, vol. 43, no 4, pp. 587-603.

VILLAÇA, F. (1998), Espaço Intra-Urbano no Brasil. São Paulo, Studio Nobel. 


\section{ABSTRACT \\ A Comparison of Social Structures in Rio de Janeiro and São Paulo}

This article presents the results of a comparative study on socio-spatial structures in Rio de Janeiro and São Paulo in 2000. We drew on data from the national Demographic Census by weighted areas to construct the Erikson, Goldthorpe, and Portocarrero (EGP) classification and the International Socio-Economic Index (ISEI), both widely used in social stratification studies. This information was then submitted to group analyses for the two cities, allowing comparison of the presence of social groups in each city. Next, using spatial statistics, we assessed the spatial distribution of the socio-economic classes and the presence of social segregation in the two metropolitan areas. The results suggest the presence of strong similarity between the social structures in the two cities, also marked by similarly intense patterns of social segregation at the metropolitan level.

Key words: social structure; occupational categories; EGP; São Paulo; Rio de Janeiro

\section{RÉSUMÉ \\ Comparaison de Structures Sociales à Rio de Janeiro et à São Paulo}

Dans cet article, on présente des résultats d'une recherche comparative sur les structures sociospatiales à Rio de Janeiro et à São Paulo en 2000. On a travaillé avec des données du recensement démographique par groupes de pondération pour construire le classement Erikson, Goldthorpe et Portocarrero (EGP), ainsi que l'indicateur international de niveau socioéconomique (SE), tous deux souvent utilisés dans des études de stratification sociale. Ces informations ont été examinées avec analyse des conglomérats pour les deux villes, permettant ainsi de comparer la présence de groupes sociaux dans les deux villes. Ensuite, à l'aide de statistiques spatiales, on a évalué la distribution spatiale des classes et la présence d'une ségrégation sociale dans ces deux espaces urbains. Les résultats suggèrent la présence d'une grande similarité entre les structures sociales dans les deux villes, marquées aussi par des modèles très intenses de ségrégation sociale.

Mots-clé: structure sociale; catégories professionnelles; EGP; São Paulo; Rio de Janeiro 